\title{
Cemented versus cementless fixation in total knee arthroplasty
}

\author{
FABRIZIO MATASSI, CHRISTIAN CARULLI, ROBERTO CIVININI, MASSIMO INNOCENTI
}

Orthopaedics Clinic, University of Florence, Italy

\begin{abstract}
The question of whether to use cemented or cementless fixation for a total knee arthroplasty (TKA) is still debated. Discouraging preliminary results of cementless TKAs have determined the worldwide use of cemented implants. However, with the development of biotechnologies and new biomaterials with high osteoconductive properties, biological fixation is now becoming an attractive option for improving the longevity of TKAs, especially in young patients.

There is no evidence in the current literature to support the use of one method of fixation. The extensive clinical experience with cemented implants gathered over the years justifies their widespread use. New randomized clinical trials are necessary to compare cementless fixation based on the new ingrowth surfaces with standard cemented implants.
\end{abstract}

Key Words: cemented, cementless, fixation, implant, knee, arthroplasty.

\section{Introduction}

The ideal fixation of a total knee arthroplasty (TKA) is still debated. The main question is whether the use of cement is more efficient than press-fit fixation in terms of ensuring durable stability. The use of cement in TKAs has been associated with excellent clinical outcomes and low rates of aseptic loosening at long-

\section{Corresponding Author:}

Fabrizio Matassi, MD

Orthopaedics Clinic, University of Florence, Italy

Largo P. Palagi 1, 50139 Florence, Italy

Phone: 055/7948287

E-mail: fabriziomatassi@gmail.com term follow-up, and it is the most widespread method of fixation in knee replacement. However, alterations of the bone/cement interface leading to osteolysis prompted orthopaedic surgeons to look for a new method of fixation that would avoid this complication, particularly in younger patients (1-3).

The basis for the use, since the mid-80s (both in Italy and in Europe), of cementless TKAs in young patients with adequate bone stock is the concept that osteoconductive component surfaces, in the presence of a very active bone metabolism, show high biological properties. Many authors proposed a "hybrid" fixation technique, consisting of a cementless femoral component and a tibial component fixed with a cemented baseplate and a press fit keel $(4,5)$. However, the demonstration, in short- and medium-term studies, of an high rate of early loosening related to micromotion led to a return to standard cemented TKAs $(6,7)$ (Tab. 1). This problem was related to the first cementless designs and the geometry of the early components, characterized by poor osteoconductive surfaces or inadequate fixation devices (pins, screws). Modern implants incorporate effective solutions (porous coatings, plasma spray, rotating platforms) able to reduce stress conditions and micromotion at the bone/metal interfaces.

\section{Cemented versus cementless TKAs}

At long-term follow-up, survival rates of up to $99 \%$ for cemented TKAs and 97\% for cementless implants have been reported $(4,8-10)$. However, the literature lacks studies that compare the two methods of fixation.

A meta-analysis of 15 studies showed a higher risk of aseptic loosening for cementless TKAs. However these studies were very heterogeneous in terms of patient cha- 
Table 1. Trend analysis of the types of TKA fixation used during the decade 2001-2010. The data shows a progressive reduction in the number of cementless and hybrid fixed implants with respect to cemented TKAs (Data from RIPO Emilia-Romagna 2000-2010).

\begin{tabular}{|ccccc|}
\hline Year & $\begin{array}{c}\text { \% Cemented } \\
\text { Implants }\end{array}$ & $\begin{array}{c}\text { \% Cementless } \\
\text { Implants }\end{array}$ & $\begin{array}{c}\text { \% Cemented Tibial } \\
\text { Components Only }\end{array}$ & Components Only \\
\hline $\mathbf{2 0 0 1}$ & 82.2 & 8.1 & 9.0 & 0.7 \\
\hline $\mathbf{2 0 0 2}$ & 78.8 & 9.0 & 11.8 & 0.4 \\
\hline $\mathbf{2 0 0 3}$ & 82.6 & 7.6 & 9.5 & 0.6 \\
\hline $\mathbf{2 0 0 4}$ & 88.0 & 7.4 & 4.0 & 0.6 \\
$\mathbf{2 0 0 5}$ & 89.9 & 6.2 & 3.3 & 0.4 \\
\hline $\mathbf{2 0 0 6}$ & 90.7 & 5.3 & 3.6 & 1.3 \\
\hline $\mathbf{2 0 0 7}$ & 91.1 & 4.5 & 3.0 & 2.4 \\
\hline $\mathbf{2 0 0 8}$ & 91.2 & 4.2 & 2.2 & 2.5 \\
\hline $\mathbf{2 0 0 9}$ & 91.5 & 4.5 & 1.5 & 1.1 \\
\hline
\end{tabular}

racteristics and knee systems implanted. Furthermore, patients were not stratified by age and level of activity. Randomized clinical trials have not demonstrated a clear superiority of cemented TKAs over cementless implants, in terms of survival rates and clinical outcomes (10).

Khaw et al. (11), in a randomized controlled study, found comparable survival rates $(95.3 \%$ for cemented TKAs versus $95.6 \%$ for cementless TKAs) in a cohort of 501 implants (227 cemented and 224 cementless) of the same design (PFC; DePuy, Warsaw, IN). Park at al. (12) analyzed clinical and radiographic results in a randomized controlled study of 50 patients undergoing a simultaneous bilateral knee replacement with implants of the same design (Nexgen; Zimmer, Warsaw, IN), which were cemented on one side and cementless on the contralateral side. The survival rate of the femoral components was found to be $100 \%$ for both implants, while the tibial plates showed a survival rate of $100 \%$ for the cemented TKAs, and $98 \%$ for the cementless TKAs. However, no significant differences were found in the clinical results (12). Other clinical studies have been performed to assess the best method of fixation, but to date no evidence exists to support the use of one over the other. Most of the case series reported in the literature present limitations, investigating heterogeneous patient samples, different implants, and first-generation porous surfaces that are not comparable in terms of bone ingrowth to the modern coatings.

\section{Cementless implants and biological fixation}

One of the main indications for using a cementless TKA is good bone quality with high metabolic activity, in order to promote biological fixation. Indeed, a younger age (under 65 years old) and an adequate bone stock are the most typical indications.

To ensure good primary stability of the implant, the bone resections must be performed accurately, avoiding any gaps between the host bone and the components. In cemented TKAs, small defects in resections can be easily filled by the cement mantle without affecting the stability (Tab. 2).

Radiostereometry analyses (RSA) have made it possi- 
Table 2. Cementless implants: critical factors.

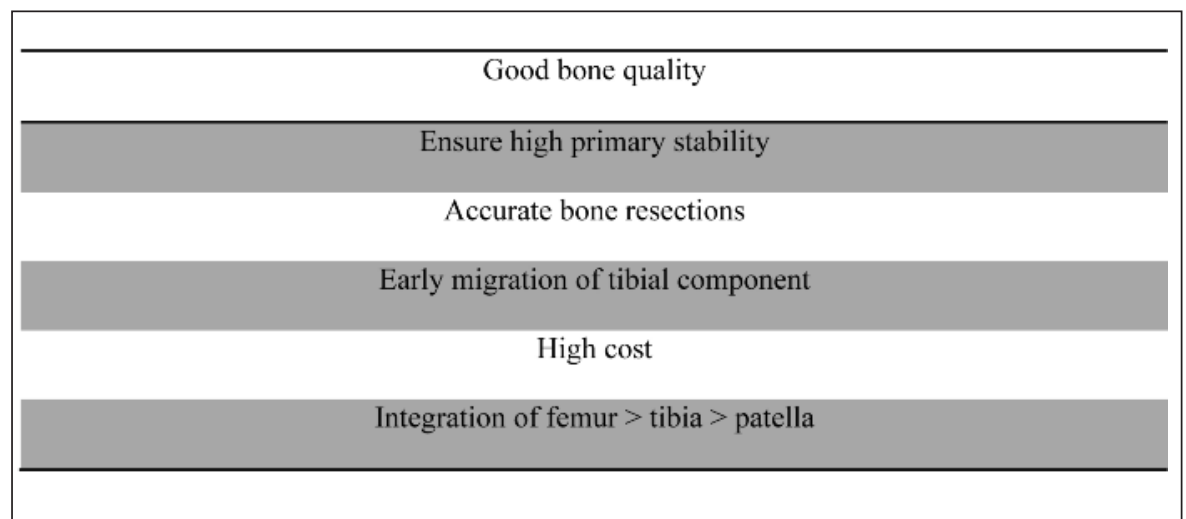

(usually the femoral component), has been proposed on the strength of the high osteoconductive properties of the modern component coatings. In a randomized controlled study, Gao et al. (14), using RSA, found similar results in terms of migration, clinical outcomes, and survival rates of 41 TKAs in young patients ( $<60$ years) undergoing knee replacement (NexGen, Zimmer, Warsaw, USA): 22 with fully cemented implants and 19 with hybrid fixated implants. Yang et al. (18), following up 235 TKAs, performed with a hybrid fixation technique and using five different knee systems, reported a survival rate of $95 \%$ at ten years, and then of $92 \%$ at fifteen years.

Cementation of the patellar component is crucial: it is now clear that cementless patellas are associated with a high risk of failure due to early loosening of the component.

\section{Cemented implants}

The extensive clinical experience with this method of fixation and the long-term studies reported in literature justify its worldwide use. Cementation allows an easier surgical technique, ensures greater primary stability as demonstrated by RSA studies, may be useful for the delivery of local antibiotics (given the diffusion of antibiotic-loaded cements), and, finally, may produce a barrier able to prevent the diffusion of wear particles over the periprosthetic bone tissue, known to be the most frequent cause of aseptic failure of knee implants. However, cemented fixation is not without drawbacks (Tab. 3).

The use of cement usually involves specific steps, which have to be carefully performed: preparation, application on the bone or component surfaces, removal of residual cement, extensive washing. All these operations take time, and thus prolong the overall surgical time.

Several studies have addressed the risks of extra-articular impingement of the cement mantle on the tibial insert, and third body wear induction by the release of 
Table 3. Cemented implants: critical factors.

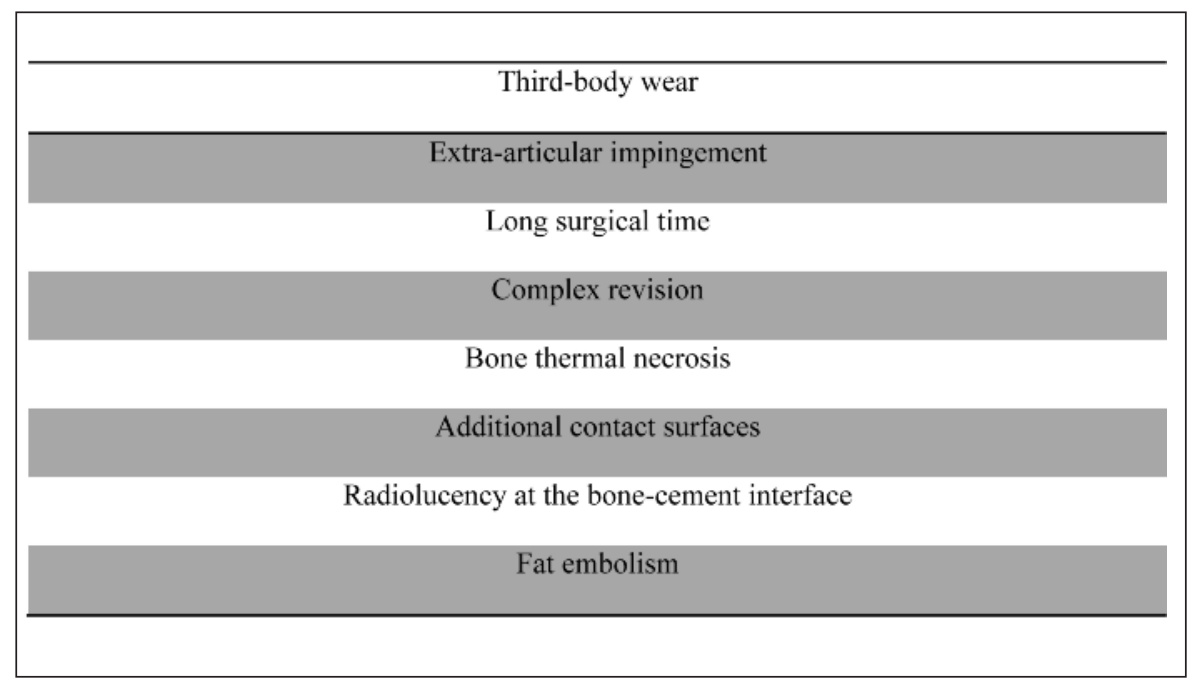

\section{Future directions}

There is still no evidence to support the use of cemented TKAs with respect to press fit implants. Longer follow-up studies are necessary to ascertain any clinical improvement of modern bioactive surfaces over traditional cemented TKAs. The study of the interactions between bone tissue and drugs active on bone metabolism is a brand-new field of interest. Even though, to date, only preliminary in vitro studies have been conducted, the results are encouraging, shoparticles in the articular space. Noble et al. (19), in their observational study of 162 revision TKAs, showed significant abrasive wear in $35 \%$ of retrieved cemented components (versus 25\% of retrieved cementless ones). However, the risk of residual particles after cementation may be prevented by thorough washing and cement removal before closing.

Moreover, thermal necrosis, which may be induced during the polymerization of the cement, carries a specific risk of tissue damage. The use of a cement mantle (generally $2 \mathrm{~mm}$ thick) introduces an additional surface (cement/bone plus cement/component) in the implant, increasing the risk of mobilization or of wear production (20). There is still a debate on which of the two interfaces is the source of failure: the cement/bone interface is generally suggested to be the critical zone. An important study of the complications of total hip arthroplasty demonstrated a high risk of fat embolism during the pressurization of the cement in the femoral canal (21). Even though there is no study of this kind in TKAs, it is reasonable to think that knee replacement may carry a similar risk. Clarke et al. (22) showed a significantly increased risk of deep venous thromboembolism in cemented TKAs with respect to cementless implants.

Finally, revisions of failed cemented TKAs are technically more demanding with respect to cementless implants, particularly on account of the frequent bone loss after removal of the components. wing a favorable effect, in terms of bone stimulation and osseointegration of knee components, of drugs generally used in bone metabolism alterations (23).

\section{References}

1. Lombardi AV Jr, Berasi CC, Berend KR. Evolution of tibial fixation in total knee arthroplasty. J Arthroplasty 2007; 22 (4 Suppl 1):25-29.

2. Naudie DD, Ammeen DJ, Engh GA, Rorabeck CH. Wear and osteolysis around total knee arthroplasty. J Am Acad Orthop Surg 2007;15:53-64.

3. O'Rourke MR, Callaghan JJ, Goetz DD, Sullivan PM, Johnston RC. Osteolysis associated with a cemented modular posterior-cruciate-substituting total knee design: five to eightyear follow-up. J Bone Joint Surg Am 2002;84:1362-1371.

4. Bassett RW. Results of 1,000 performance knees: cementless versus cemented fixation. J Arthroplasty 1998;13:409-413.

5. Nolan JF, Bucknill TM. Aggressive granulomatosis from polyethylene failure in an uncemented knee replacement. J Bone Joint Surg Br 1992;74:23-24.

6. Berry DJ, Wold LE, Rand JA. Extensive osteolysis around an aseptic, stable, uncemented total knee replacement. Clin Orthop Relat Res 1993;(293):204-207.

7. Rendiconto Attività R.I.P.O Registro Regionale di Implantologia Protesica Ortopedica Emilia Romagna 2000-2010.

8. Rand JA. Cement or cementless fixation in total arthroplasty? Clin Orthop Relat Res 1991; (273):52-62.

9. McCaskie AW, Deehan DJ, Green TP, et al. Randomised, prospective study comparing cemented and cementless total knee replacement: results of press-fit condylar total knee replacement at five years. J Bone Joint Surg Br 1998;80:971975.

10. Gandhi R, Tsvetkov D, Davey JR, Mahomed NN. Survival and clinical function of cemented and uncemented prostheses in total knee replacement: a meta-analysis. J Bone Joint Surg Br 2009;91:889-895.

11. Khaw FM, Kirk LM, Morris RW, Gregg PJ. A randomised, 
controlled trial of cemented versus cementless press-fit condylar total knee replacement. Ten-year survival analysis. J Bone Joint Surg Br 2002;84:658-666.

12. Park JW, Kim YH. Simultaneous cemented and cementless total knee replacement in the same patients: a prospective comparison of long-term outcomes using an identical design of NexGen prosthesis. J Bone Joint Surg Br 2011;93:14791486.

13. Nilsson KG, Kärrholm J, Carlsson L, Dalén T. Hydroxyapatite coating versus cemented fixation of the tibial component in total knee arthroplasty: prospective randomized comparison of hydroxyapatite-coated and cemented tibial components with 5-year follow-up using radiostereometry. J Arthroplasty 1999;14:9-20.

14. Gao F, Henricson A, Nilsson KG. Cemented versus uncemented fixation of the femoral component of the NexGen CR total knee replacement in patients younger than 60 years: a prospective randomised controlled RSA study. Knee 2009; 16:200-206.

15. Sorrells RB, Voorhorst PE, Murphy JA, Bauschka MP, Greenwald AS. Uncemented rotating-platform total knee replacement: a five to twelve-year follow-up study. J Bone Joint Surg Am 2004;86-A:2156-2162.

16. Ali MS, Mangaleshkar SR. Uncemented rotating-platform total knee arthroplasty: a 4-year to 12-year follow-up. J Arthroplasty 2006;21:80-84.
17. Sharma S, Nicol F, Hullin MG, McCreath SW. Long-term results of the uncemented low contact stress total knee replacement in patients with rheumatoid arthritis. J Bone Joint Surg Br 2005;87:1077-1080.

18. Yang JH, Yoon JR, Oh CH, Kim TS. Hybrid component fixation in total knee arthroplasty: minimum of 10-year follow-up study. J Arthroplasty 2012; 27:1111-1118.

19. Noble PC, Conditt MA, Thompson MT, Stein JA, Kreuzer S, Parsley BS, Mathis KB. Extraarticular abrasive wear in cemented and cementless total knee arthroplasty. Clin Orthop Relat Res 2003;(416):120-128.

20. Bert JM, McShane M. Is it necessary to cement the tibial stem in cemented total knee arthroplasty? Clin Orthop Relat Res 1998;(356):73-78.

21. McCaskie AW, Barnes MR, Lin E, Harper WM, Gregg PJ. Cement pressurisation during hip replacement. J Bone Joint Surg Br 1997;79:379-384.

22. Clarke MT, Green JS, Harper WM, Gregg PJ. Cement as a risk factor for deep-vein thrombosis. Comparison of cemented TKR, uncemented TKR and cemented THR. J Bone Joint Surg Br 1998;80:611-613.

23. Carulli C, Civinini R, Matassi F, Villano M, Innocenti M. The use of antiosteoporotic drugs in total knee arthroplasty. Aging Clin Exp Res 2011; 23 (2 Suppl):38-39. 